\title{
Pleuritic chest pain in a postpartum woman
}

J. S. MACGREGOR, A. W. WU \& H. R. RUBIN

Department of Medicine, University of California, San Francisco

\section{CASE REPORT}

A 26-year-old woman, gravida 3, para 2, came to the emergency room complaining of pleuritic chest pain and shortness of breath. Twelve days prior, she had a vaginal delivery induced by pitocin. Her recovery was uneventful until the morning of admission when she awoke with bilateral pleuritic chest pain radiating to her shoulders, accompanied by dyspnea at rest. She also described mid-epigastric pain with nausea and anorexia. She denied fever, chills, rhinorrhea, cough, sputum production, haemoptysis, vomiting, change in bowel habits, constipation, diarrhea, or leg pain. The patient did not smoke, did not use oral contraceptives, and was on no medications. She had never had abdominal or gynaecologic surgery.

She appeared comfortable with normal respirations. The heart rate was 80 beats $\min ^{-1}$, the respiratory rate $16 \mathrm{~min}^{-1}$, the blood pressure $120 / 90 \mathrm{~mm}^{-1}$ of mercury, and the temperature $37 \cdot 1^{\circ} \mathrm{C}$. The chest was clear. Cardiac auscultation revealed regular rhythm with no murmur, gallop, click or rub. Jugular venous pressure was normal. The abdomen was flat and nondistended with normal bowel sounds. There was mild upper abdominal tenderness with no guarding or rebound. No organomegaly was detected.

Arterial blood gas determination showed a $\mathrm{pH}$ of $7.42, \mathrm{pO}_{2}$ of $93 \mathrm{~mm}$ of mercury, $\mathrm{pCO}_{2}$ of $37 \mathrm{~mm}$ of mercury, with a carbon dioxide content of $25 \mathrm{mmolli}^{-1}$. A chest radiograph showed no infiltrates or effusions, but demonstrated a large amount of subdiaphragmatic, extraluminal air (Fig. 1).

Further history revealed that on the night before her illness began, the patient had engaged in sexual intercourse for the first time in 4 months, including oral-vaginal insufflation.

In view of the possiblity of a ruptured viscus, a surgical consultation was obtained. Subsequent laboratory tests included haemoglobin 139 grams litre $^{-1}$ leukocyte count of $7.1 \times 10^{9}$ litre $^{-1}$ with 0.64 segmented neutrophils and no bands. Electrolytes, trasaminases, and amylase were normal. KUB showed a non-specific bowel gas pattern and confirmed the presence of free subdiaphragmatic air.

The patient was admitted to the hospital for observation. Chest X-rays over the next $48 \mathrm{~h}$ showed diminution of the amount of free air. Her pleuritic chest pain improved, and she was discharged.

Correspondence: John S. MacGregor, M.D., Box 0124, M-1186, 505 Parnassus Avenue, University of California, San Francisco, San Francisco, CA 94143, U.S.A. 


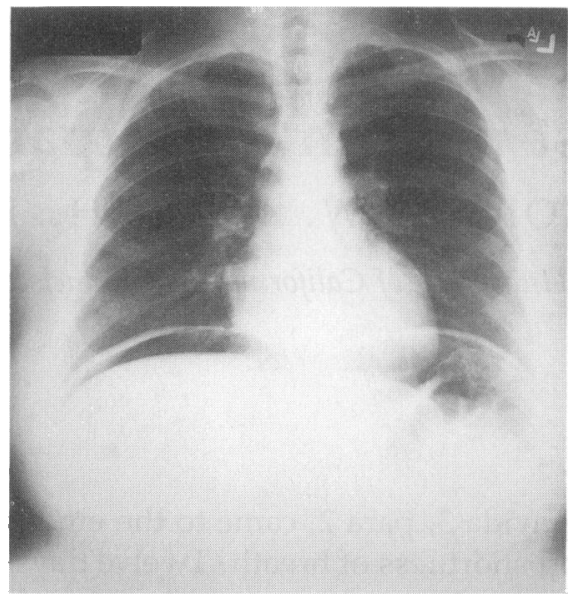

Fig. 1 Chest radiograph demonstrating a large amount of extraluminal subdiaphragmatic air

\section{DISCUSSION}

Pneumoperitoneum due to oral-vaginal insufflation is infrequently reported (Wright, 1959; Freeman, 1970; Spaulding \& Gallup, 1979; Christiansen et alo $\overrightarrow{0}$ 1980; Elam \& Ray, 1986; Smally, 1989), and in most cases has occurred following. hysterectomy, apparently due to a rent in the vaginal cuff and direct entry of ais into the peritoneum (Spaulding \& Gallup 1979; Christiansen et al., 1980; Elam \& Ray, 1986). Three cases have been reported in the absence of previous hysterectomy (Wright, 1959; Freeman, 1970; Smally, 1989). The proposed mechanism is passage of air into the uterus through the cervical os, then to the peritoneum through the fallopian tubes. This patient may have had a partially open cervical os due to her recent delivery. In most cases, patients have been hospitalized for observation to exclude the possibility of a ruptured viscus. In one previous report, the patient was discharged from the emergency room without admission to the hospital (Smally, 1989).

Diaphragmatic irritation due to pneumoperitonem should be considered in the differential diagnosis of pleuritic chest pain. When pneumoperitoneum is discovered without a history of recent surgery or instrumentation, and without signs of peritonitis, vaginal insufflation should be considered as a possible cause, even without previous hysterectomy. If such a history is obtained, in the setting of a benign abdominal exam and normal laboratory studies, expectant management may suffice, sparing the patient unnecessary antibiotics or surgery.

Primary care physicians and obstetricians may wish to advise their postpartum patients to avoid sexual practices involving oral-vaginal insufflation. 


\section{REFERENCES}

Christiansen W. C., Danzl D. F. \& McGee H. J. (1980) Pneumoperitoneum following vaginal insufflation and coitus. Annals of Emergency Medicine 9(9), 480-2

Elam A. L., \& Ray V. G. (1986) Sexually related trauma: A review. Annals of Emergency Medicine 15, 576-584.

Freeman R. K. (1970) Pneumoperitoneum from oral genital insufflation. Obstetrics and Gynecology 36, $162-164$.

Smally A. J. (1989) Referred shoulder pain in a sexually active woman. Hospital Practice July 15, 62-64. Spaulding L. B. \& Gallup D. G. (1979) Pneumoperitoneum after hysterectomy. Journal of the American Medical Association 241, 825.

Wright A. R. (1959) Spontaneous penumoperitoneum. Archives of Surgeny 78, 500-502. 